\title{
Weak antilocalization and conductance fluctuation in a single crystalline Bi nanowire
}

\section{Citation}

Kim, Jeongmin, Seunghyun Lee, Yuri M. Brovman, MinGin Kim, Philip Kim, and Wooyoung Lee. 2014. "Weak Antilocalization and Conductance Fluctuation in a Single Crystalline Bi Nanowire." In Applied Physics Letters 104, no. 4: 043105. doi:10.1063/1.4863421.

\section{Published Version}

doi:10.1063/1.4863421

\section{Permanent link}

http://nrs.harvard.edu/urn-3:HUL.InstRepos:22557381

\section{Terms of Use}

This article was downloaded from Harvard University's DASH repository, and is made available under the terms and conditions applicable to Other Posted Material, as set forth at http:// nrs.harvard.edu/urn-3:HUL.InstRepos:dash.current.terms-of-use\#LAA

\section{Share Your Story}

The Harvard community has made this article openly available.

Please share how this access benefits you. Submit a story.

\section{Accessibility}




\title{
Weak antilocalization and conductance fluctuation in a single crystalline $\mathrm{Bi}$ nanowire
}

\author{
Jeongmin Kim, ${ }^{1}$ Seunghyun Lee, ${ }^{1}$ Yuri M. Brovman, ${ }^{2}$ MinGin Kim, ${ }^{1}$ Philip Kim, ${ }^{2, a)}$ \\ and Wooyoung Lee Le) $^{1, a)}$ \\ ${ }^{1}$ Department of Materials Science and Engineering Yonsei University, 134 Shinchon, Seoul 120-749, \\ South Korea \\ ${ }^{2}$ Department of Physics, Columbia University, New York, New York 10027, USA
}

(Received 30 September 2013; accepted 14 January 2014; published online 28 January 2014; publisher error corrected 6 February 2014)

\begin{abstract}
We present the low temperature transport properties of an individual single-crystalline Bi nanowire grown by the on-film formation of nanowire method. The temperature dependent resistance and magnetoresistance of $\mathrm{Bi}$ nanowires were investigated. The phase coherence length was obtained from the fluctuation pattern of the magnetoresistance below $40 \mathrm{~K}$ using universal conductance fluctuation theory. The obtained temperature dependence of phase coherence length and the fluctuation amplitude indicates that the transport of electrons shows 2-dimensional characteristics originating from the surface states. The temperature dependence of the coherence length derived from the weak antilocalization effect using the Hikami-Larkin-Nagaoka model is consistent with that from the universal conductance fluctuations theory. (C) 2014 AIP Publishing LLC. [http://dx.doi.org/10.1063/1.4863421]
\end{abstract}

Semimetal bismuth has been widely employed for the study of electrical quantum effects, especially for the enhancement of thermoelectric performance due to quantum confinement. $^{1-4}$ The low carrier effective mass, $m^{*}$, and long mean free path, $l$, of Bi result in high electrical mobility. For Bi nanowires $(\mathrm{NW})$, the small carrier concentration, $n$, and the band overlap energy leads to semimetal-semiconductor (SMSC) transition as the diameter of the wires decreases. ${ }^{1,2}$ This SMSC transition is predicted for Bi nanowires with diameters less than $30 \mathrm{~nm}$, depending on the crystal orientation, and is concurrent with the enhancement of the Seebeck coefficient. ${ }^{3,4}$ Studies of magnetic transport phenomenon to analyze the surface states of Bi nanowires show quantum oscillations, such as Shubnikov-de Hass ( $\mathrm{SdH}$ ) and Aharonov-Bohm (AB), superimposed on magnetoresistance (MR). ${ }^{5,6}$ These quantum oscillations in $\mathrm{Bi}$ nanowires suggest significant contribution from the 2-dimensional (2D) surface states in smaller diameter $(d<50 \mathrm{~nm})$ Bi nanowires where the surface to volume ratio become drastically different from the bulk. ${ }^{7,8}$ Recent studies also revealed that the surface induced effect becomes more pronounced as the SMSC transition becomes apparent for the Bi nanowires with smaller diameters. ${ }^{9}$

Although the properties of low dimensional Bi nanowire, including low carrier concentration, long mean free path, low effective carrier mass, and anisotropic Fermi pockets, have been investigated, the quantitative analysis of properties related to the electron interference effects has not been reported in $\mathrm{Bi}$ nanowires with the diameters smaller than $35 \mathrm{~nm}$, the regime where the SMSC transition and the surface states significantly change the electronic properties of nanowires. For a mesoscopic system with disorder and strong spin-orbit coupling, the phase coherent magneto-transport at low temperatures result in the appearance of weak antilocalization (WAL) and universal

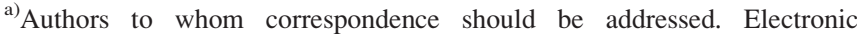
addresses: wooyoung@yonsei.ac.kr and pk2015@columbia.edu
}

conductance fluctuations (UCF) effects. ${ }^{10-12}$ These phenomena are affected by the phase-coherence length, $l_{\phi}$, defined as the length where phase-coherent transport is preserved. Therefore, WAL and UCF are commonly used in determining the phase-coherence length of a material system. ${ }^{13-15}$

In this paper, we report the WAL and UCF in a singlecrystalline $\mathrm{Bi}$ nanowire grown by on-film formation of nanowire (OFF-ON) method. ${ }^{16}$ The phase coherence length of $\mathrm{Bi}$ nanowire was analyzed using magnetoresistance by the Hikami-Larkin-Nagaoka (HLN) model and UCF theory. ${ }^{17,18}$ The obtained temperature dependence of the phase coherence length indicates that the $2 \mathrm{D}$ transport behavior due to the surface states of $\mathrm{Bi}$ nanowire dominate the observed bulk transport characteristics.

Single-crystalline $\mathrm{Bi}$ nanowires were grown using OFF-ON method (Fig. 1(a)). ${ }^{16}$ Bi films with a $44 \mathrm{~nm}$ thickness were deposited on thermally oxidized Si (100) substrate using a custom-made sputter deposition system. Since the diameter of the nanowires is dependent on the grain size of the film, liquid nitrogen cooling system was used to obtain small diameter Bi nanowires close to $30 \mathrm{~nm} .{ }^{19}$ The sputtering system was evacuated to $10^{-7}$ Torr prior to deposition, and the vacuum was maintained during sputtering under 2 mTorr Ar environment for $10 \mathrm{~s}$. The growth rate of the Bi thin film was $44 \AA / \mathrm{s}$. The Bi thin films were subsequently placed in a custom-made vacuum furnace for thermal annealing to grow nanowires by OFF-ON method (Fig. 1(b)). Annealing was performed in a vacuum of $10^{-7}$ Torr at $250^{\circ} \mathrm{C}$ for $5 \mathrm{~h}$. The growth direction of the nanowire is [100], which is vertical to the transmission electron microscope (TEM) image shown in Figure 1(c), and the electron diffraction (ED) pattern shows that the nanowire is a perfect single-crystalline (Fig. 1(d)). ${ }^{20}$ A thermally oxidized Si (100) substrate was patterned with a grid of markers by photolithography and lift-off process before $\mathrm{Bi}$ nanowire dispersion. The $\mathrm{Bi}$ nanowires were dispersed by direct contact of grown nanowires to the $\mathrm{Si}$ 

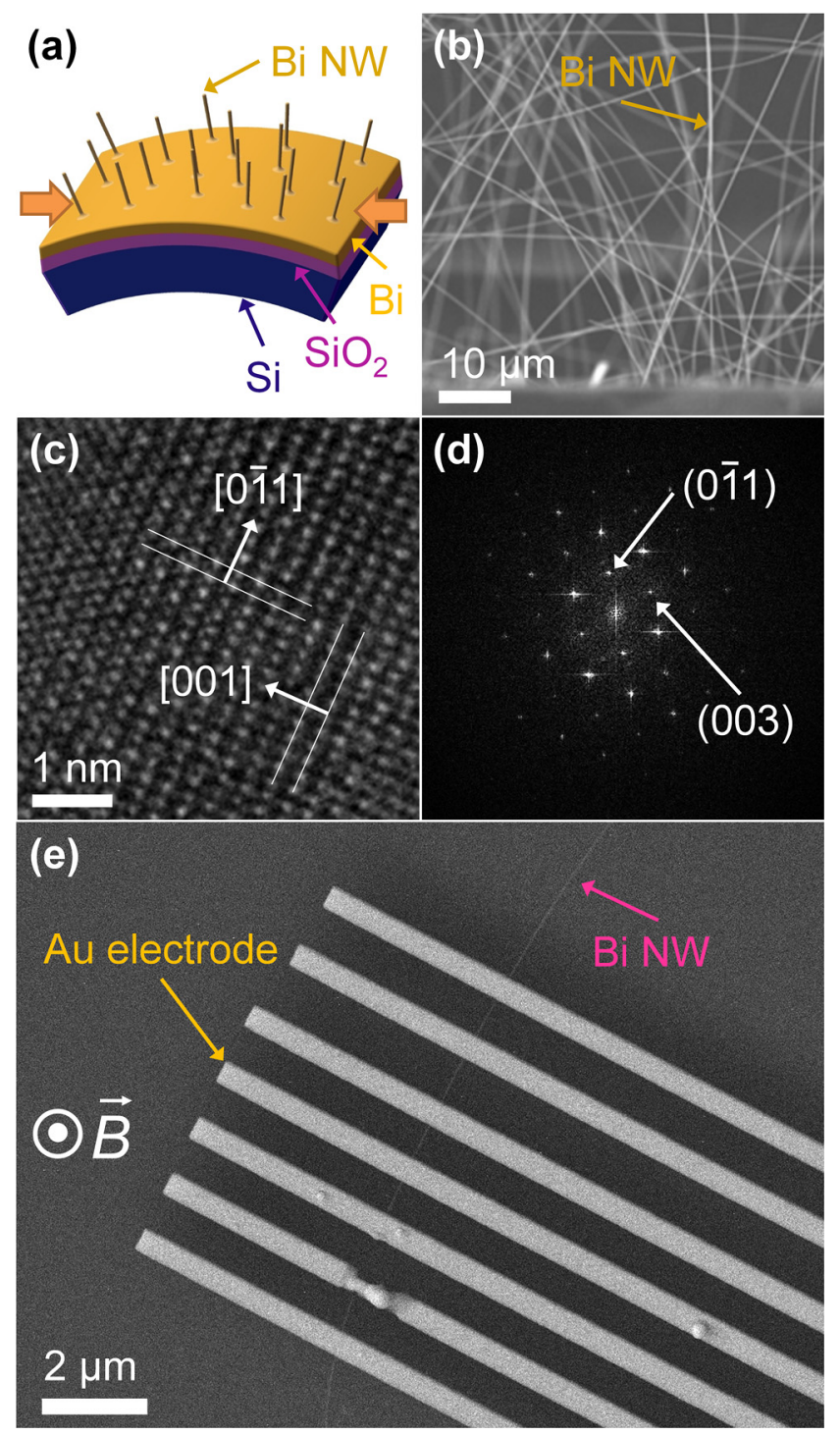

FIG. 1. (a) A schematic representation of the growth of Bi nanowires by the OFF-ON method: Bi nanowires are grown to release thermal stress originating from thermal expansion mismatch between the $\mathrm{SiO}_{2}$ and $\mathrm{Bi}$ thin film. (b) A scanning electron microscope (SEM) image of $\mathrm{Bi}$ nanowires grown on a Bi thin film. (c) A high-resolution TEM image of a typical Bi nanowire. The sample is sliced perpendicular to growth direction. (d) TEM electron diffraction pattern of a $\mathrm{Bi}$ nanowire. The [100] zone axis indicates the growth direction. (e) A SEM image of a typical 4-probe device based on the individual Bi nanowire geometry.

substrate. The combination of electron-beam lithography and a lift-off process was utilized to fabricate inner micron-scaled $\mathrm{Cr} / \mathrm{Au}(2 \mathrm{~nm} / 200 \mathrm{~nm})$ electrodes. Plasma etching system was used to remove the oxide layer from the outer surface of the nanowires before the deposition of electrodes (Fig. 1(e)). ${ }^{21,22}$ The etching and deposition of electrodes were done in-situ without breaking the vacuum in order to prevent further formation of the oxide layer. All measurements were done in a helium cryostat equipped with a $9 \mathrm{~T}$ magnet at the temperature range from $1.85 \mathrm{~K}$ to $300 \mathrm{~K}$. The magnetic field was perpendicular to the growth direction of the wires. The magnetoresistance was measured by a lock-in technique with an AC bias current of $100 \mathrm{nA}$. All measurements were done by the 4-terminal method in order to remove the contribution of contact resistance.

Figure 2(a) shows the temperature dependence of the resistance of three individual single-crystalline $\mathrm{Bi}$ nanowires (a)

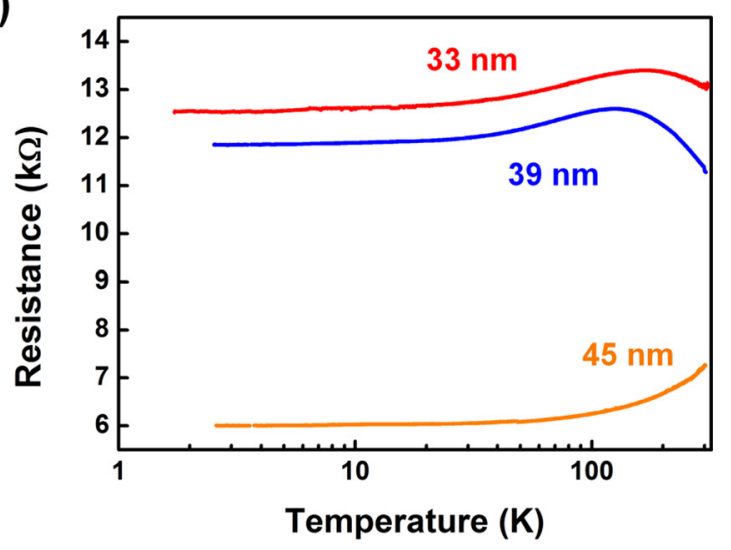

(b)

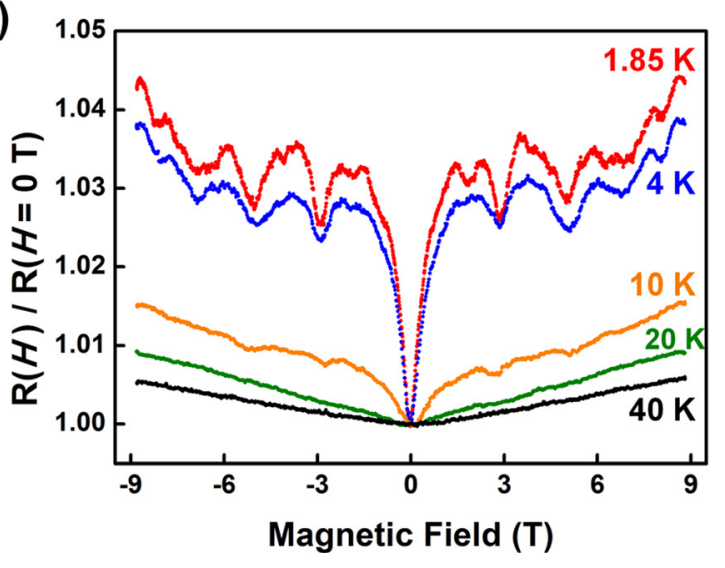

FIG. 2. (a) Temperature dependence of resistance for three different diameters 33, 39, and $45 \mathrm{~nm}$ at zero magnetic field. (b) Magnetoresistance as a function of magnetic field, normalized to the resistance at zero magnetic field, for the $33 \mathrm{~nm}$ diameter Bi nanowire.

with different diameters, 45, 39, and $33 \mathrm{~nm}$, respectively. Since the carrier concentration of $\mathrm{Bi}\left(\sim 3 \times 10^{17} \mathrm{~cm}^{-3}\right)$ is much smaller compared to normal metals, the temperature, $T$, dependent resistance, $R(T)$, measured in these nanowires are determined by the competition between mobility and carrier concentration. ${ }^{6}$ We observe $R(T)$ of the $45 \mathrm{~nm}$ Bi nanowire monotonically decreases with decreasing $T$. For thinner nanowires, $R(T)$ exhibits a non-monotonic temperature dependence, i.e., $R(T)$ first increases as $T$ decreases followed by decreasing $R(T)$ for decreasing $T<100 \mathrm{~K}$. Similar diameter dependent $R(T)$ behaviors were observed in Bi nanowires in a previous study, ${ }^{1}$ where the non-monotonic dependence in small diameter nanowires is attributed to the competition between $T$ dependent carrier-acoustic phonon scattering and the carrier non-specular surface scattering due to the classical size effect. In this study, we focus on the smallest diameter $(33 \mathrm{~nm})$ nanowire in the low temperature regime where the phase coherent quantum transport becomes apparent.

Figure 2(b) shows the magnetoresistance of individual Bi nanowire with $33 \mathrm{~nm}$ diameter at temperatures between 1.85 and $40 \mathrm{~K}$, normalized to the resistance at zero magnetic field at the same temperature range. Magnetoresistance shows two physical phenomena related to the phase coherence length $l_{\phi}$. The first is the narrow valley originating from WAL near the zero magnetic field. ${ }^{10,14}$ Usually, WAL occurs when the strong spin-orbit interaction reverses the sign of the resistance correction in the weak localization (WL) phenomena. Without a strong spin-orbit coupling, the WL effect 
can be identified by the increase of resistance near the zero magnetic field because of the conductance correction by electron localization. In WAL, the additional phase shift arising from the spin-orbit interaction leads to the constructive interference of time-reversal pair of electron waves scattered by impurities, which in turn reduces the resistance around zero magnetic field. The second is the UCF which originates from the change of interference of the time-reversal pair of electron waves in a mesoscopic system, i.e., the size of system is not too different from $l_{\phi}$, when the phase of the waves shifts according to the enclosed magnetic flux in the various closed loops of electron trajectories. ${ }^{11,15}$ These two effects are based on diffusive transport phenomena due to elastic scattering, and therefore can only be observed in low-temperature range where the inelastic scattering of phonons and electrons are frozen out. For Bi nanowires, the aforementioned effects were prominent below $10 \mathrm{~K}$ and were used to determine the phase coherence length, $l_{\phi}$, of the material system. ${ }^{14,15,23,24}$

For a more detailed analysis of the electron interference with UCF, the corresponding conductance fluctuations $\delta G$ was separated from the magnetoresistance (Fig. 2(b)). The magnetoresistance was normalized to the conductance quantum $e^{2} / h$ after the classical contribution $G_{0}$ was eliminated. The classical MR is proportional to the fundamental quantity $\omega_{\mathrm{c}} \tau$, where $\omega_{\mathrm{c}}$ and $\tau$ are the cyclotron frequency and relaxation time, respectively. ${ }^{5}$ The cyclotron frequency $\omega_{\mathrm{c}}$ is proportional to the square of the magnetic field $B .{ }^{16}$ To extract the classical contribution, the quadratic fitting of measured MR was used. Figure 3(a) shows the conductance fluctuation at $1.85,4$, and $10 \mathrm{~K}$, and the decrease of amplitude can be observed as the temperature increases. The amplitude of $\delta G$ with a magnitude of one-tenth of $e^{2} / h$ at $1.85 \mathrm{~K}$ agrees well with the correlation equation of the amplitude, $\delta G$,

$$
\delta G \propto\left(l_{\phi} / L\right)^{3 / 2},
$$

since the channel length, $L$, is relatively longer compared to the phase coherence length $l_{\phi}{ }^{11}$

For the quantitative analysis of the magnitude of $\delta G$, root mean square (rms) of the conductance fluctuation, $\operatorname{rms}(\delta G)$, which is defined by $\sqrt{\left\langle\delta G^{2}\right\rangle}$, is used. Here, $\langle\ldots\rangle$ expresses the ensemble average. Figure 3(b) shows the temperature dependence of $\operatorname{rms}(\delta G)$ in $\log$ scale. The value increases as temperature decreases. The temperature dependence of the conductance fluctuation from 1.85 to $20 \mathrm{~K}$ is linear and fitted to be $T^{-0.77}$ as shown by the dash line. We will discuss the temperature dependence of $\operatorname{rms}(\delta G)$ later after the analysis of the temperature dependence of the phase coherence length $l_{\phi}$. Another important quantity that can be derived from $\delta G$ is the correlation field $B_{c}$, which can be calculated through the autocorrelation function $F(\Delta B)=\langle\delta G(B+\Delta B) \delta G(B)\rangle \cdot{ }^{18} B_{c}$ is defined as the full width at half maximum of the autocorrelation function $F\left(B_{c}\right)=\frac{1}{2} F(0)$. From the $B_{c}$ value calculated from $\delta G(B)$, it can be seen that $B_{c}$ linearly decreases proportional to $T^{0.43}$ as the temperature decreases from $40 \mathrm{~K}$ to $1.85 \mathrm{~K}$ (Fig. 3(c)). The temperature dependence of $l_{\phi}$ can be determined from the relation of $B_{C}$ and the phase coherence length, $l_{\phi}, l_{\phi} \approx \Phi_{0} / B_{c} d$, where $\Phi_{0}$ and $d$ are the magnetic flux quantum, $\Phi_{0}=h / e,^{21,25}$ and diameter, respectively.
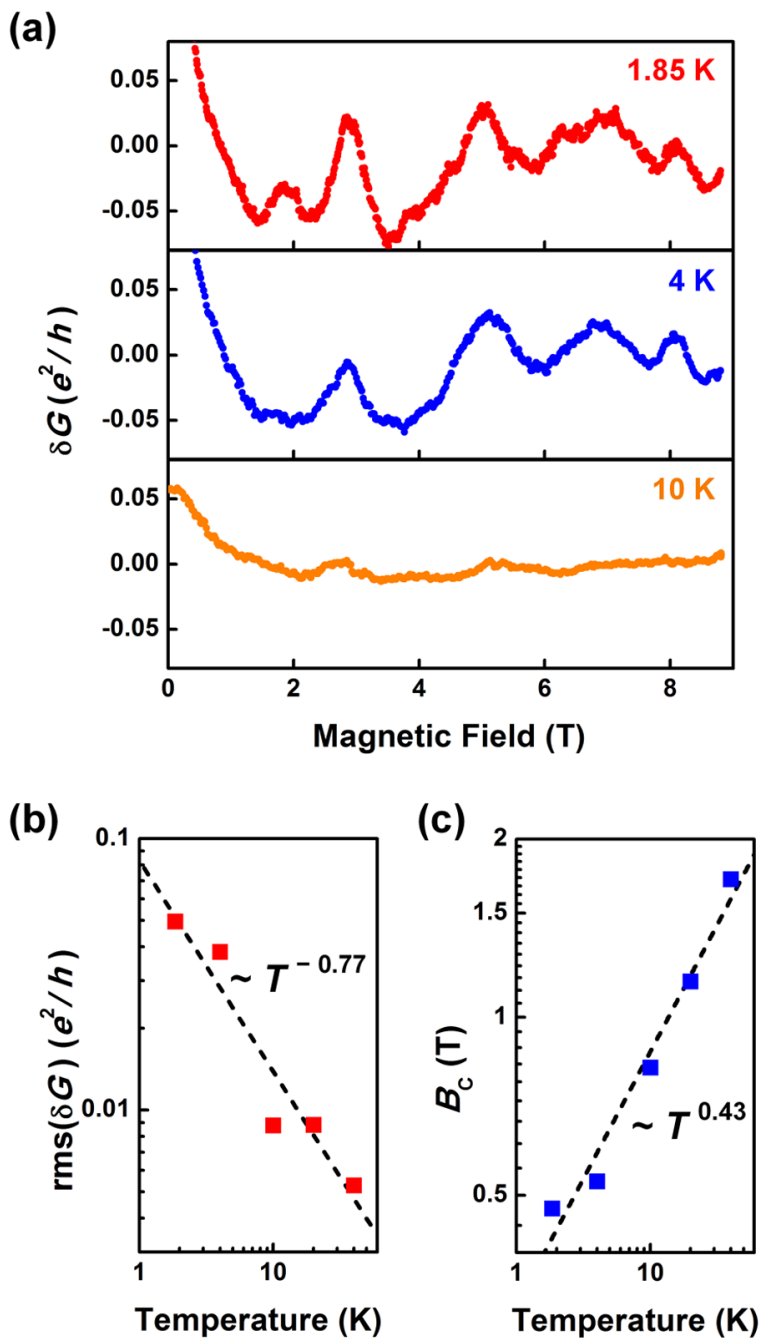

FIG. 3. (a) Conductance fluctuations for $33 \mathrm{~nm}$ diameter as function of magnetic field at $1.85,4$, and $10 \mathrm{~K}$. (b) Rms of the conductance fluctuations plotted against temperature. The temperature dependence of the conductance fluctuation is fitted to be $T^{-0.77}$, as shown by the dashed line. (c) Correlation field as a function of temperature. The dashed line represents the $T^{0.43}$ dependence.

A direct relation between $B_{c}$ and $l_{\phi}$ allows us to find the temperature dependent phase coherence length. Figure 4(a) shows the obtained temperature dependence of the coherence length $l_{\phi}$ using UCF analysis, which increases with decreasing temperature proportional to $T^{-0.43}$. From the temperature dependence of $l_{\phi}$, we can obtain the dimensionality of the system. Theoretically, the coherence length is proportional to $T^{-1 / 3}, T^{-1 / 2}$, and $T^{-3 / 4}$ for the one, two, and three-dimensional system, respectively. ${ }^{26}$ Previous studies reported that Bi nanowire with the diameter of $100 \mathrm{~nm}$ has one-dimensional system $^{12}$ compared to three-dimensional bulk characteristics shown in $400 \mathrm{~nm}$ diameter. ${ }^{21,22}$ At the large diameter range, the 2D surface state effect is veiled due to the small surface to volume ratio and large bulk electrons compared to the surface carrier density. ${ }^{12}$ However, the obtained exponent of -0.43 reflects that the system is a hybridization of one and two dimensions. The fact that this exponent is closer to the two-dimensional system value -0.5 suggests that the surface state of Bi plays a significant role in transport. Furthermore, the fitting which regards the saturation of $l_{\phi}$ at low temperature shows perfect linear dependence and the exponent of 
(a)

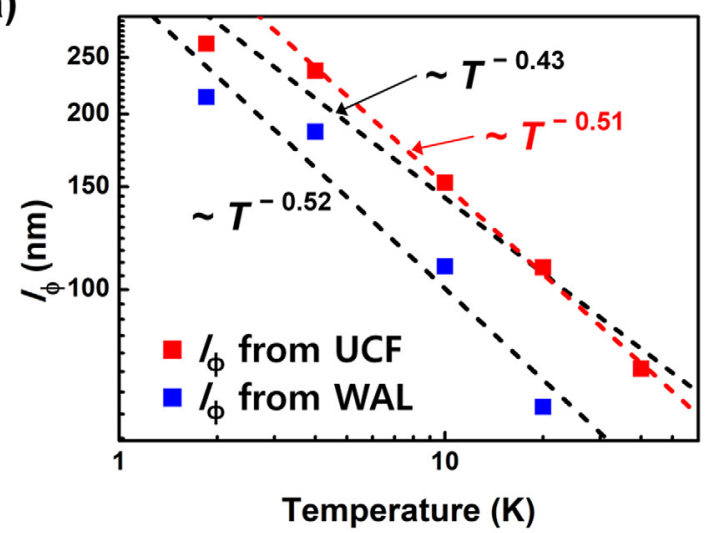

(b)

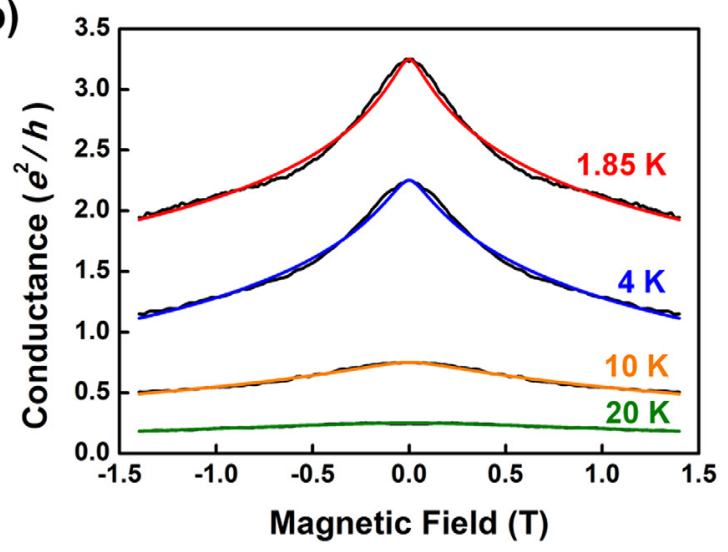

FIG. 4. (a) Coherence lengths for the $33 \mathrm{~nm}$ diameter nanowire as a function of temperature obtained by the UCF theory (red) and the HNL model (blue). The black dashed lines show that the temperature dependence of the coherence lengths are fitted to be $T^{-0.43}$ and $T^{-0.52}$, respectively. The red dashed line represents the linear fitting result which regards the saturation of the coherence length at low temperatures. (b) Magneto-conductance around zero magnetic field shows a weak antilocalization effect. The colored curves present the fitting results by the HLN model. The y-axis scale is taken from the data obtained at $20 \mathrm{~K}$. The other curves are incrementally shifted upward by $e^{2} / h$ for clarity of presentation.

$-0.51 .^{15}$ In Bi thin films and Bi nanowires below $50 \mathrm{~nm}$, surface state effect appears on bulk characteristics due to its large surface to volume ratio., ${ }^{7,8}$ The surface induced carrier concentration originating from the surface state was found to be $n_{s}=5 \times 10^{12} \mathrm{~cm}^{-2}$. The estimated carrier concentration of the $33 \mathrm{~nm}$ diameter nanowire was calculated to be $6.0 \times 10^{18} \mathrm{~cm}^{-3}$, based on the relation of the nanowire carrier concentration $2 n_{s} / r$, where $r$ is the radius of the nanowire. The surface induced carrier concentrations of the nanowires are significantly large relative to that of the one-dimensional system, and, as a result, the system is dominated by two-dimensional characteristics. The quantum transport parameters with variation of temperature are listed in Table I.

We now discuss the temperature dependence of $\operatorname{rms}(\delta G) \sim T^{-0.77}$ observed above. Since the estimated $l_{\phi}$ in all temperature range of our experiment is less than $500 \mathrm{~nm}$, which is much less than the channel length $L=1 \mu \mathrm{m}$ in our device, we expect Eq. (1) to hold for the amplitude fluctuation for $\delta G$. From the previous discussion, we can approximate $l_{\phi} \sim T^{-1 / 2}$, therefore, we expect $\operatorname{rms}(\delta G) \sim l_{\phi}^{3 / 2} \sim T^{-3 / 4}$. The temperature dependence of $\operatorname{rms}(\delta G)$ observed in the $\mathrm{Bi}$ NW is proportional to $T^{-0.77}$, which is close to the theoretically expected value from the UCF theory, ${ }^{18}$ and reaffirms
TABLE I. Quantum transport properties obtained from UCF and WAL effects in $33 \mathrm{~nm}$ Bi nanowire.

\begin{tabular}{lcccc}
\hline \hline & $\begin{array}{c}\mathrm{UCF} \\
\mathrm{T}(\mathrm{K})\end{array}$ & $\mathrm{UCF}$ & $\mathrm{UCF}$ & $\mathrm{WAL}$ \\
\hline 1.85 & 0.050 & 0.475 & 264.0 & 214.0 \\
4 & 0.038 & 0.528 & 237.4 & 186.5 \\
10 & 0.009 & 0.822 & 152.5 & 109.5 \\
20 & 0.009 & 1.149 & 109.1 & 62.9 \\
40 & 0.004 & 1.712 & 73.2 & \\
\hline \hline
\end{tabular}

that the dimensionality of the system is dominated by $2 \mathrm{D}$ characteristics.

The WAL of MR in weak magnetic field range ( -1.4 to 1.4 T) is similar to the UCF. Since WAL is sensitive to the phase coherence, the temperature dependence of $l_{\phi}$ can be estimated from the background of magneto-conductance change, $\delta G_{\mathrm{WAL}}(B)=G(B)-G(0)$. In the HLN theory, ${ }^{17}$ $\delta G_{\mathrm{WAL}}$ can be related to $l_{\phi}$ via the phase coherence magnetic field $B_{\phi}=\frac{\hbar}{4 e l_{\phi}^{2}}$, using the equation

$$
\delta G_{\mathrm{WAL}}(B)=-\alpha \frac{e^{2}}{2 \pi^{2} \hbar}\left[\psi\left(\frac{1}{2}+\frac{B_{\phi}}{B}\right)-\ln \left(\frac{B_{\phi}}{B}\right)\right],
$$

where $\alpha$ and $\psi$ are the fitting parameter and the digamma function, respectively. Fitting Eq. (2) for $B_{\phi}$ for the curves of $\delta G_{\mathrm{WAL}}(B)$ at different temperature (Fig. 4(b)), we can then find the temperature dependent $l_{\phi}$. Figure 4(a) shows $l_{\phi}$ obtained from the WAL analysis compared to the previously obtained $l_{\phi}$ from the UCF analysis. We find that $l_{\phi}$ from the WAL analysis shows $T^{-0.52}$ dependence, which is consistent with that obtained from the UCF theory.

In summary, the magnetoresistance of an individual single-crystalline $\mathrm{Bi}$ nanowire grown by the OFF-ON method was measured. The observed dip near zero magnetic field and the fluctuation with magnetic field sweep originate from WAL effect and UCF, respectively. The temperature dependence of the phase coherence length obtained from the UCF theory confirmed that the transport of carriers shows $2 \mathrm{D}$ characteristics that originates from the surface states of Bi nanowires. The exponential coefficient from the temperature dependence of the coherence length derived from the WAL effect using HLN model is consistent with the value obtained from the UCF theory. Our investigations demonstrate that the surface states of $\mathrm{Bi}$ nanowires dominate the bulk transport characteristics in the diameter range close to $30 \mathrm{~nm}$.

This work was supported by the Agency for Defense Development (ADD-10-70-07-03), the Priority Research Centers Program (No. 2009-0093823), and the Pioneer Research Center Program (No. 2013008070) through the National Research Foundation of Korea (NRF), and the U.S. National Science Foundation (DMR-1122594).

${ }^{1}$ J. Heremans, C. M. Thrush, Y. M. Lin, S. Cronin, Z. Zhang, M. S. Dresselhaus, and J. F. Mansfield, Phys. Rev. B 61, 2921-2930 (2000).

${ }^{2}$ Z. Zhang, X. Sun, M. S. Dresselhaus, J. Y. Ying, and J. Heremans, Phys. Rev. B 61, 4850-4861 (2000).

${ }^{3}$ L. D. Hicks and M. S. Dresselhaus, Phys. Rev. B 47, 16631-16634 (1993). 
${ }^{4}$ Y. M. Lin, X. Sun, and M. S. Dresselhaus, Phys. Rev. B 62, 4610-4623 (2000).

${ }^{5}$ F. Y. Yang, K. Liu, K. Hong, D. H. Reich, P. C. Searson, and C. L. Chien, Science 284, 1335-1337 (1999).

${ }^{6}$ T. E. Huber, A. Nikolaeva, D. Gitsu, L. Konopko, C. A. Foss, Jr., and M. J. Graf, Appl. Phys. Lett. 84, 1326-1328 (2004).

${ }^{7}$ A. Nikolaeva, D. Gitsu, L. Konopko, M. J. Graf, and T. E. Huber, Phys. Rev. B 77, 075332 (2008).

${ }^{8}$ P. Hofmann, Prog. Surf. Sci. 81, 191-245 (2006).

${ }^{9}$ T. E. Huber, A. Adeyeye, A. Nikolaeva, L. Konopko, R. C. Johnson, and M. J. Graf, Phys. Rev. B 83, 235414 (2011).

${ }^{10}$ C. W. J. Beenakker and H. van Houten, Solid State Physics: Advances in Research and Applications (Academic Press, New York, 1992), vol. 44, p. 1.

${ }^{11} \mathrm{H}$. Ibach and H. Lüth, Solid State Physics: An Introduction to Principles of Materials Science, (Springer, New York, 2009), p. 278.

${ }^{12}$ N. Marcano, S. Sangiao, M. Plaza, A. F. Pacheco, R. Cordoba, M. C. Sanchez, L. Morellon, M. R. Ibarra, and J. M. De Teresa, Appl. Phys. Lett. 96, 082110 (2010).

${ }^{13}$ Z. Li, T. Chen, H. Pan, F. Song, B. Wang, J. Han, Y. Qin, X. Wang, R. Zhang, J. Wan, D. Xing, and G. Wang, Sci. Rep. 2, 595 (2012).

${ }^{14}$ S. Matsuo, T. Koyama, K. Shimamura, T. Arakawa, Y. Nishihara, D. Chiba, K. Kobayashi, and T. Ono, Phys. Rev. B 85, 075440 (2012).
${ }^{15}$ Ch. Blömers, Th. Schäpers, T. Richter, T. Calarco, H. Lüth, and M. Marso, Appl. Phys. Lett. 92, 132101 (2008).

${ }^{16}$ W. Shim, J. Ham, K. Lee, W. Jeung, M. Johnson, and W. Lee, Nano Lett. 9, 18-22 (2009).

${ }^{17}$ S. Hikami, A. Larkin, and Y. Nagaoka, Prog. Theor. Phys. 63, 707 (1980).

${ }^{18}$ P. Lee, A. Stone, and H. Fukuyama, Phys. Rev. B 35, 1039-1070 (1987).

${ }^{19}$ S. Lee, J. Ham, K. Jeon, J. Noh, and W. Lee, Nanotechnology 21, 405701 (2010).

${ }^{20}$ J. Roh, K. Hippalgaonkar, J. Ham, R. Chen, M. Li, P. Ercius, A. Majumdar, W. Kim, and W. Lee, ACS Nano 5, 3954-3960 (2011).

${ }^{21}$ W. Shim, J. Ham, J. Kim, and W. Lee, Appl. Phys. Lett. 95, 232107 (2009).

${ }^{22}$ K. Lee, S. Lee, S. N. Holmes, J. Ham, W. Lee, and C. H. W. Barnes, Phys. Rev. B 82, 245310 (2010).

${ }^{23}$ J. Chen, H. J. Qin, F. Yang, J. Liu, T. Guan, F. M. Qu, G. H. Zhang, J. R. Shi, X. C. Xie, C. L. Yang, K. H. Wu, Y. Q. Li, and L. Lu, Phys. Rev. Lett. 105, 176602 (2010).

${ }^{24}$ H. T. He, G. Wang, T. Zhang, I. K. Sou, G. K. L. Wong, J. N. Wang, H. Z. Lu, S. Q. Shen, and F. C. Zhang, Phys. Rev. Lett. 106, 166805 (2011).

${ }^{25}$ C. W. J. Beenakker and H. Houten, Phys. Rev. B 37, 6544-6546 (1988).

${ }^{26}$ B. L. Altshuler, A. G. Aronov, and D. E. Khmelnitskii, J. Phys. C: Solid State Phys. 15, 7367-7386 (1982). 